\title{
POLFEL - A Free Electron Laser in Poland
}

\author{
Ryszard S. Romaniuk* \\ Institute of Electronic Systems, Warsaw University of Technology, Nowowiejska 15/19, 00-665 Warszawa
}

Received August 31, 2009; accepted September 3, 2009; published September 30, 2009

\begin{abstract}
The free electron laser in Poland - POLFEL is a coherent light source of the $\mathrm{IV}^{\text {th }}$ generation, characterized by very short pulses in the range of $10-100 \mathrm{fs}$, of big power $0,2 \mathrm{GW}$ and UV wavelength of $27 \mathrm{~nm}$ of average power $1 \mathrm{~W}$, with an effective high power third harmonic of $9 \mathrm{~nm}$. The laser consists of a linear superconducting accelerator $100 \mathrm{~m}$ in length, undulator and experimental lines. It generates monochromatic and coherent radiation and can be tuned from the THz range to UV. The linac works in quasi-CW or real-CW mode. It is planned by XFELPoland consortium as a part of the ESFRI priority EuroFEL infrastructure collaboration network, part of the ERA.
\end{abstract}

Recent developments of technology enabled a number of new fields of basic and applied research. One can mention such areas as: work on fundamental laws of physics, new materials engineering, more efficient semiconductors, quantum optics and chemistry, structural biology, biomedicine with targeted imaging, photo and radiotherapy, new medicines. Research methods in distant fields have common features. Research needs enormous sets of data and immense processing power for simulations and analysis. Researched objects vary from nano, via pico to femtometers. The objects are cells, molecules, micella, atoms, nuclei, elementary particles. Time spans of concern, for observed dynamic processes like chemical reactions, are also femto and attoseconds. These advanced research methods require large infrastructures including accelerators of high energy particles, synchrotrons, free electron lasers, as well as, classical ones, but of high power and/or high intensity.

Poland never has never had even a single infrastructure of this kind. This is a serious obstacle in carrying out one's own advanced research in a number of the above mentioned fields. The national budget for research has been far too small to dream of such undertakings. Now, the chance for this country is double: there are relevant funds and projects as well as appropriate research and technical institutions to undertake the effort. The obtained European structural funds for 2007-13 include 1.3bln $€$ for research infrastructures. These resources are enough to build a few big experiments in Poland fit to the ERA scale.

The Polish research community of synchrotron radiation, FELs, attosecond and HP-HI lasers, organized in a number of consortia, among them XFEL-Poland, participate actively in many European research networks,

*E-mail: rrom@ise.pw.edu.pl collaborations and projects like: ELI, HIPER, LaserLab, E-XFEL, EuroFEL, to mention a few. Access to the knowledge and experience of the whole European synchrotron and laser communities is available. This is a solid gate and background to building our own complementary laser infrastructures, using the newest, yet checked, technologies and avoiding too expensive, and out of reach, development phase of the project like building prototypes for early system commissioning.

The design of POLFEL was constructed in this way so as to take advantage of these chances - political, financial and technical - and establish, for the first time in history, a research project of the European size in Poland.

\section{FEL components and technical data}

FEL consists of an electron injector, linac, undulator, electron beam dump (or recovery) and photon lines. Future FELs will use superconducting (SC) injectors with $\mathrm{Nb}$ cavity. Conventional RF gun consists of a warm $\mathrm{Cu}$ cavity with a photocathode of $\mathrm{Cs}_{2} \mathrm{Te}$. The photocathode is excited with a UV pulse of $\lambda=266 \mathrm{~nm}$, which is the $4^{\text {th }}$ harmonic from a Nd:YAG laser. A single laser pulse, together with a presence of $\mathrm{HV}$ at a cathode, causes emission of an electron cloud of $\mathrm{Q}_{\mathrm{e}} \approx 1 \mathrm{nC}$ charge. The pulse repetition rate is confined to $f_{\text {gun }}=1 \mathrm{kHz}$ by the thermal stability of the RF gun, caused by heat release in $\mathrm{Cu}$. The $\mathrm{Cu}$ and the following chain of $\mathrm{Nb}$ cavities are powered by a HP klystron or a klistrode (IOT) of $\mathrm{P}_{\mathrm{RF}}=10 \mathrm{MW}$.

The electron cloud from the RF gun has to be magnetically compressed, spatially and energetically, after some pre-acceleration, to a coherent bunch. The $120 \mathrm{MeV}$ injector gives electron bunches of $\mathrm{L}_{\mathrm{b}} \approx 1 \mathrm{~mm}$ in length. The demands for the compact electron bunch of quality stem from the requirements on the resulting photon pulse from the laser, and are as follows: high pulse current $\mathrm{I}_{\mathrm{e}} \approx 1 \div 10 \mathrm{kA}$; average beam current $\mathrm{I}_{\mathrm{b}}=1 \mathrm{~mA}$; beam power $\mathrm{P}_{\mathrm{b}} \approx 350 \mathrm{~kW}$; beam density $\mathrm{dP}_{\mathrm{b}} / \mathrm{ds} \approx 3,5 \cdot 10^{11} \mathrm{~W} / \mathrm{cm}^{2}$, bunch separation $\mathrm{t}_{\mathrm{sb}} \approx 1 \mu \mathrm{s}$; short spatial, including longitudinal $\mathrm{L}_{\mathrm{b}}<100 \mu \mathrm{m}$ and transverse $\mathrm{D}_{\mathrm{b}}=10 \mu \mathrm{m}$, as well as temporal $\mathrm{T}_{\mathrm{b}}<1 \mathrm{ps}$ dimensions; correlation of position-energy of electrons in the bunch. The compressor of the beam consists of a magnetic chicane (4 $\mathrm{H}$ type dipole electromagnets of strong $\mathrm{B}_{\mathrm{H}} \approx 0,5 \mathrm{~T}$ and homogeneous $\mathrm{s}_{\mathrm{H}}=300 \mathrm{~mm}$ field causing $\mathrm{U}$ bend of the $\mathrm{e}^{-}$path), correction coils for each magnet, and 
three beam refocusing quadrupoles. The resulting $\mathrm{e}^{-}$ bunches from the first compressor, positioned after the injector, are very dense, short, coherent energetically $\Delta \mathrm{E}<\mathrm{E}_{\text {coh }}$, and of small transverse emittance $\varepsilon<1 \mu \mathrm{rad}$. The second compressor (and beam corrector) is placed just before the undulator and the $\mathrm{H}$ field parameters of chicane dipoles are optimized for the incoming bunch energy $\mathrm{E}_{\mathrm{b}} \approx 0,5 \div 1 \mathrm{GeV}$. The input energy of the undulator depends on the active length of the linac, i.e. the number of crio-modules.

The linac uses a well established, and used widely around the globe, superconducting niobium TESLA cavities, organized in 9 cell structures (resonators) of $\mathrm{L}_{\mathrm{c}} \approx 1 \mathrm{~m}$ and $\mathrm{L}_{\mathrm{cc}} \approx 1,2 \mathrm{~m}$, with 8 ones in a single crio-module (cold mass). The purity of $\mathrm{Nb}$ is $\mathrm{RRR}>300$. Resonant frequency of the cavity is $\mathrm{f}_{\mathrm{c}} \approx 1,3 \mathrm{GHz}$ (L-band). Each of 9 cell resonators is supplied by $\mathrm{RF}$ power $\mathrm{P}_{\mathrm{RF}} \approx 100 \div 300 \mathrm{~kW}$ via rectangular waveguides filled with $\mathrm{SF}_{6}$. Field intensity in the cell is $\mathrm{E}_{\mathrm{RF}} \approx 20 \div 35 \mathrm{MV} / \mathrm{m}$. The fundamental mode is TM010. The unloaded/loaded $Q$ of the structure is respectively $\mathrm{Q}_{\mathrm{ul}}=10^{10}, \mathrm{Q}_{\mathrm{ld}}>10^{7}$. The work temperature of the structure is $\mathrm{T}_{\mathrm{C}}=1,8 \mathrm{~K}$ obtained in three levels, via $40 \div 80 \mathrm{~K}$, and $5 \div 8 \mathrm{~K}$ shields to the state of superfluid helium. The criogenic load of the structure is $\mathrm{P}_{\mathrm{cr}} \approx 3 \mathrm{~W}$.

Each crio-module contains: 8 TESLA structures; 8 HP $\mathrm{RF}$ waveguide/coaxial line criogenic fundamental mode couplers -FMC; 8 two-function, slow-fast, mechanical tuners; 16 high order mode couplers -HOMC; HOM absorber; ion vacuum pump; correcting magnet and quadrupole lens; beam position monitor -BPM; helium vessel; cold mass package for reference positioning of components and thermal insulation; criogenic cabling for measurements, diagnostics and control.

The resonator is subject to Lorentz force detuning (LFD) and microphonics (MP). LFD is a reaction of the $\mathrm{Nb}$ SC resonator, of the average wall thickness $\mathrm{d}_{\mathrm{w}} \approx 2,5 \div 3 \mathrm{~mm}$, thus, not very stiff, to filling with HP EM RF field. The resonators are stiffened with stiffening rings and collars and sealed titanium chambers for superfluid He. The LFD, via the change in cavity dimensions $L_{c}$ and $D_{c}$, results in the change in the resonant frequency $f_{c}$. Typical value of the frequency dynamic detuning $\Delta \mathrm{f}_{\mathrm{LFD}}$ is equal to the cavity $3 \mathrm{~dB}$ bandwidth $\Delta \mathrm{f}_{\mathrm{c}} \approx 200 \div 300 \mathrm{~Hz}$.

Each resonator is filled with an accelerating field, via a power coupler, and then works in a pulse or CW mode. The coupler consists of: waveguide to coax transition; two vacuum - microwave windows, one ambient temperature $\mathrm{T}_{\mathrm{w} 1}=\mathrm{T}_{\mathrm{a}}$, the second cold $\mathrm{T}_{\mathrm{w} 2}=70 \mathrm{~K}$; thermal insulation components from $\mathrm{T}_{\mathrm{a}}$ to $\mathrm{T}_{\mathrm{c}}=1,8 \mathrm{~K}$; Nb coaxial head ended with antenna of controlled coupling to the HP field of the FM in the accelerating structure.

Beam loading and acceleration in the structure excites longitudinal and/or transverse higher order modes (HOM) to propagate. The HOM have higher frequencies than the fundamental mode (FM), thus, can be filtered out by a low pass filter. The HOM distort bunches via a disturbance to the FM field distribution. A HOM filter is an antenna situated on both ends of the structure and strongly coupled to higher frequencies (not coupled to FM).

Each structure is equipped with a slow and fast, mechanical tuner, with a piezoelectric converter (voltageshift $\mathrm{V}-\Delta \mathrm{L}_{\mathrm{c}}$ ). The resonance $\mathrm{f}_{\mathrm{ci}}$ of each $\mathrm{i}$-th structure is individually adjusted to the accelerating frequency $f_{a}$ by squeezing or stretching the cavity of $\Delta \mathrm{L}_{\mathrm{cs}}$. Slow tuning, with reaction time $t_{s t}=1 \mathrm{~min}$, for static pre-detuning purpose, is done mechanically in clams within typical margins $\Delta \mathrm{L}_{\mathrm{cs}} \approx \pm 1 \mathrm{~mm}$ for $\mathrm{L}_{\mathrm{c}} \approx 1 \mathrm{~m}$, which corresponds to $\Delta \mathrm{f}_{\mathrm{cs}} \approx \pm 300 \mathrm{kHz}$. Fast tuning, for Lorentz force detuning, and microphonics compensation, as well as dynamic predetuning, is done dynamically, during cavity filling, with the reaction time $t_{\mathrm{dLFD}} \approx 1 \mathrm{~ms}$ and frequency tuning range $\Delta \mathrm{f}_{\mathrm{cd}} \approx \pm 0,5 \mathrm{kHz}$. The value of $\Delta \mathrm{f}_{\mathrm{cd}}$ is comparable to $\Delta \mathrm{f}_{\mathrm{c}}$, which guarantees precise tuning tracking of the cavities.

HP RF supply system provides microwave power of $\mathrm{f}_{\mathrm{acc}} \approx 1,3 \mathrm{GHz}$ to fill the accelerating cavities with the EM filed and then maintain the field intensity in the range of $\mathrm{E}_{\mathrm{acc}} \approx 25 \div 35 \mathrm{MV} / \mathrm{m}$. The HP RF system works in a pulse and CW modes and consists of: power amplifiers, modulators, inductive output tubes (IOT), HV HP supply units, control amplifiers, protection and safety circuits, power distribution via transmission waveguides with $\mathrm{Y}$ and $\mathrm{T}$ couplers, isolators and circulators from IOT to cavities, and auxiliary components.

The accelerator control system (ACS) consists of: low level RF (LLRF), including reference time distribution from the master oscillator, measurement, synchronization and fast control via FF/FB modes; slow control (SCt); $\mathrm{e}^{-}$ beam diagnostics, interlock and experiment diagnostics. The LLRF system stabilizes the field in accelerating cavities, generates the accelerator clock and synchronizes all equipment with this clock. The SCt controls all FEL infrastructure including: vacuum, cryogenics, gases, HVs, power supplies, ambient parameters. The diagnostics localizes potential causes of system misbehaviour, failures and all unexpected departures from standardized procedures, defined by state machines (SM). Diagnostics has to be nondependent from other systems and highly reliable, similarly to interlocks. The interlock system traces many threshold values in FEL and reacts accordingly when the threshold is crossed, including full stop of the machine when the work parameter values are critical. All these subsystems are managed by SCADA industrial supervisory control and data acquisition.

The LLRF system stabilizes the HP RF field using direct, predictive or adaptive feed forward (FF), feedback (FB) or a mixed method FF+FB. The FF compensates deterministic, systematic errors while FB nondeterministic ones (noise). The changes in the 
accelerating field amplitude and phase, as an error signal, are fed back in the loop to the modulator of the klystron (or IOT) causing relevant changes in the HP generator output. The requirements on the HP RF field stability are: $10^{-4}$ in amplitude and $10^{-1}$ in phase in degrees. The RF field in the cavity is probed by an antenna, downconverted to $\mathrm{f}_{\mathrm{IF}}$, sampled by ADC, processed for error, converted to analog in DAC and input to vector modulator of the klystron.

Cryogenics for a single cold mass requires $\mathrm{P}_{1 \text { cold }}=50 \mathrm{~W}$ of cold power at $T_{C}=2 \mathrm{~K}$. Accumulated output cold power from the cold plant for injector, six cold masses and helium transport lines in POLFEL is estimated for $\mathrm{P}_{\text {coldd }} \approx 500 \mathrm{~W}$. Superfluid helium II is extremely penetrable and has nearly ideal heat conduction. This requires maintaining low pressure in the He installation piping in cryo-modules $\mathrm{P}_{\mathrm{HeCM}}=30 \mathrm{mbar}$. The cold plant produces $\mathrm{He}$ in different thermodynamic states and in the following temperatures, required by successive thermal shields in the cold-mass: $\mathrm{T}_{1 \mathrm{TS}} \approx 40 \div 80 \mathrm{~K}, \mathrm{~T}_{2 \mathrm{Ts}} \approx 5 \div 8 \mathrm{~K}, \mathrm{~T}_{\mathrm{c}}=1,9 \mathrm{~K}$. The radiation shields work at $\mathrm{T}_{\mathrm{RS}}=4,5 \mathrm{~K}$. The cold plant consists of: warm He compressors, heat exchangers, turbine decompressors and Jule-Thomson choking walves. Cold compressors in the $\mathrm{He}$ return lines avoid flows of large gas volumes at ambient temperature.

The high quality UHV vacuum line, made of 316LN steel, oil-less, dustless, at $\mathrm{p}<10^{-8} \mathrm{mbar}$, embraces RF gun, $\mathrm{e}^{-}$beam path and photon paths. Part of the vacuum lines are cold $\mathrm{T}_{\mathrm{c}}=2 \mathrm{~K}$ in the cavities, and part ore warm $\mathrm{T}_{\mathrm{a}}=20^{\circ} \mathrm{C}$ in electron optics, undulators and photon diagnostics. The initial out-pumping is done by turbomolecular pumps and then the vacuum is maintained by geter-ion and sorption pumps.

Undulators, which are in series, periodic, linear stack of magnetic dipoles of opposite field direction, are tailored to the $\mathrm{e}^{-}$energy. There are used in FEL planar hybrid undulator sections with steady NdFeB magnets. The undulator parameters are: slit $\mathrm{s}_{\text {und }}=5 \div 15 \mathrm{~mm}$, period $\mathrm{p}_{\text {und }}=5 \div 30 \mathrm{~mm}$, length $\mathrm{l}_{\text {und }}=2 \div 10 \mathrm{~m}$, magnetic induction $B_{\text {und }}=0,5 T$. The $e^{-}$beam is monitored and focused inside the undulator, between sections, by quadrupole magnets. The undulator is protected against stray synchrotron radiation generated inside.

There are two main mechanisms to generate THz-IRV-UV-X coherent photon beam by the $\mathrm{e}^{-}$beam in the undulator: SASE - self amplified spontaneous emission or HGHG - high gain harmonic generation and enhanced by SEED - seeding with a laser beam. SASE means that there is a self-modulating interaction between a train of highly relativistic $\mathrm{e}^{-}$bunches and photons generated by the bunches. The bunches are self-sliced to flat microbunches of high density of charge, and the process is a function of the undulator length. A sliced bunch generates photons coherently and intensely as a point source.
The $\mathrm{e}^{-}$beam goes to an energy recovery circuit, instead to a dump, in a future FEL.

The distributed diagnostics of the whole length of the $\mathrm{e}^{-}$beam consists of: toroids, beam position monitor BPM, optical transition radiation OTR, beam lost monitor BLM, beam inhibit system BIS, transversal deflecting structure TDS, laser heater for regeneration of the photocathode, collimators and quadrupoles.

The photon beam diagnostics measures (and reacts with a control feedback): pulse energy; beam intensity; wave spectra; direction, divergence and beam waist, transverse dimensions of photon beam; pulse duration; distribution of the wave front; polarization. Photon beam parameters are essential for the experiments.

The FEL is housed in a radiation shielded tunnel with a very stable, one piece floor along the whole length of the electron and photon beams. The total power requirements for a FEL of the POLFEL size are $\mathrm{P}_{\text {tel }} \approx 3 \mathrm{MW}$.

\section{Research and Technical Scope of the FEL Project}

FEL enables new technologies and applications:

- research, ultra high field science, attosecond laser science, high energy beam facility, photon physics, twophoton absorption, pump-probe and photo thermal beam deflection PTBD spectroscopy, band edge spectroscopy, - material processing and engineering, laser ablation and deposition, welding, adiabatic nano-melting, material structure modification, nanostructures formation, new semiconductors and glasses, meta-materials, formation of corrosion resistant layers, lithography, organic crystals, - biomedical and environmental, THz generation for imaging, lens-less diffractive imaging, photon bio-probes, molecules imaging and spectroscopy, scalpels, lidars,

- chemical, fs scale time-resolved (TR) observations of chemical reactions, TR spectroscopy and holography, energy levels in strongly charged ions, molecule probing.

\section{EuroFEL Network and other relations of POLFEL}

EuroFEL consortium is a network of complementary European FEL infrastructures, a part of ERA, accepted by ESFRI. POLFEL is closely related to E-XFEL and its predecessor FLASH. The essential solutions are based on superconducting RF TESLA technology. The members of the EuroFEL, apart from the already mentioned ones, are: Fermi at Elettra, Bessy FEL, SPARX at ENEA/INFN, 4GLS at Daresbury, Orsay FEL, MAX-IV. The biggest FEL commissioned in 2009 is LCLS working in SLAC. On the domestic scene, POLFEL is related to the Nat.Ctr. of Synchrotron Radiation, under construction in Kraków.

\section{References}

[1] XFEL Poland Consortium [xfel.pl]; POLFEL [polfel.pl]

[2] E-XFEL [xfel.eu]; EuroFEL [eurofel.eu]

[3] FLASH [flash.desy.de]; TESLA [tesla.desy.de]

[4] POLFEL, Project description, IPJ, Świerk 2008/09 